\section{Psychometric evaluation of a unified Portuguese-language version of the Body Shape Questionnaire in female university students}

\section{Avaliação psicométrica de uma versão unificada em língua portuguesa do Body Shape Questionnaire para uso em estudantes universitárias}

\section{Evaluación psicométrica de la versión unificada en lengua portuguesa del Body Shape Questionnaire en estudiantes femeninas universitarias}

Wanderson Roberto Silva 1

David Costa 2

Filipa Pimenta 2

João Maroco 2

Juliana Alvares Duarte Bonini Campos 1

\begin{abstract}
The objectives of this study were to develop a unified Portuguese-language version, for use in Brazil and Portugal, of the Body Shape Questionnaire (BSQ) and to estimate its validity, reliability, and internal consistency in Brazilian and Portuguese female university students. Confirmatory factor analysis was performed using both original (34-item) and shortened (8-item) versions. The model's fit was assessed with $\chi^{2} / d f$, CFI, NFI, and RMSEA. Concurrent and convergent validity were assessed. Reliability was estimated through internal consistency and composite reliability $(\alpha)$. Transnational invariance of the BSQ was tested using multi-group analysis. The original 32-item model was refined to present a better fit and adequate validity and reliability. The shortened model was stable in both independent samples and in transnational samples (Brazil and Portugal). The use of this unified version is recommended for the assessment of body shape concerns in both Brazilian and Portuguese college students.
\end{abstract}

Psychometrics; Body Image; Reproducibility of Results;

Surveys and Questionnaires

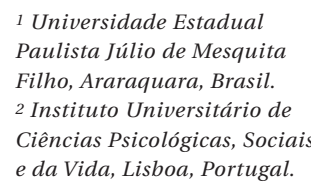

1 Universidade Estadual Paulista Júlio de Mesquita Filho, Araraquara, Brasil. 2 Instituto Universitário de Ciências Psicológicas, Sociais e da Vida, Lisboa, Portugal.

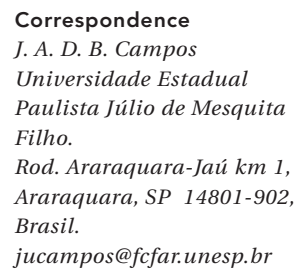




\section{Introduction}

Body image is considered a conscious representation of the body that relies largely on visual information and understands the sizes and shapes of body parts and the body as a whole 1,2. Body image reflects how people perceive their bodies 3,4 and is influenced by age, sex and gender, culture, and medical conditions 5 . The scientific literature defines body image as a multi-dimensional construct with two main components: (1) the evaluation/affect, which regards the person's judgment of his/her body, leading to satisfaction or dissatisfaction, and the emotional responses to that evaluation; and (2) the investment in and importance of appearance, jointly reflecting the person's internalized appearance standards through cognitions and behaviors targeting body image 6,7.

Body image can be interpreted positively or negatively according to individuals' feelings about their own bodies 7 . Negative self-assessment of the body can be associated with eating disorders, such as anorexia and bulimia, as well as with obesity 8,9,10,11. Research on body image dissatisfaction has focused on multiple aspects, including concerns over body shape 12 , weight gain 13 , current weight 14 , physical appearance 15 , muscle mass, and definition 16 . Since perception of one's body impacts psychological functioning and quality of life 5 , and young women are more susceptible to body image dissatisfaction than any other group 17,18, the assessment of body issues is important and should be performed 19, especially among young women.

One way of assessing body image is to use psychometric instruments 7,12,20,21,22, which address different aspects of body image. Concerns over body shape are an important aspect of body image that can be estimated by the Body Shape Questionnaire (BSQ) 12,23.

The BSQ was developed by Cooper et al. 12 to evaluate feelings of fatness after eating, exaggerated concern over body shape, and public embarrassment and avoidance among women. The instrument was originally proposed in English and displayed a one-factor structure (body shape concern), with 34 self-answered items on a 6-point Likert response scale (ranging from never to always). The items were based on the obtained from a sample of British women with and without eating disorders.

The BSQ has been used in multiple studies and has several versions adapted to populations/samples from different countries, including Colombia 24, England 25, France 26, Turkey 27, Mexico 28, Spain 29,30, Sweden 31, Brazil 15, and Portugal 13. Different versions are now available in Portuguese, proposed independently by Brazilian 15,32 and Portuguese researchers 13. The Brazilian version had its psychometric properties tested on a sample of college students 33 , while the Portuguese version was evaluated with a sample of middle-aged women 13 , and both presented good psychometric properties.

Considering the diversity of versions of the BSQ available in different languages, it is important to highlight the growing interest in transnational studies in the literature. Such studies seek to provide unified and consistent versions that can be used in countries with different cultures. Transnational studies that use a single, consistent version are important, since a single version of the questionnaire allows comparison between different situations by increasing the study's representativeness. This technique can increase the accuracy of comparisons between studies, the efficacy of the discussions, and the amount of interactions between researchers. It is essential to test the validity, reliability, and stability of any new version in order to demonstrate its utility in different cultural contexts 34,35 .

Since the BSQ is commonly used in both Brazilian and Portuguese studies 13,36,37, and there are some language differences between the Portuguese versions used in these two Portuguesespeaking countries, the current study aimed to present a unified Portuguese-language version of the BSQ that can be understood as easily in both Brazil and Portugal. The study's other goal was to estimate the psychometric properties and transnational invariance of the questionnaire when applied to a sample of both Brazilian and Portuguese university students.

\section{Methods}

\section{Participants}

Estimation of the minimum sample size was based on Kim 38 , who suggests that minimum sample size can be calculated using the model's significance level, power, and degrees of freedom. Considering alpha $=5 \%$, power $=80 \%$, and 527 degrees of freedom, the minimum sample size for this study was estimated at 67 individuals for each country (Brazil and Portugal). We also considered a loss rate of $30 \%$, so the minimum sample size was extended to 96 subjects for each country. Finally, because of the need to assess the factor structure's stability in independent samples, the estimated sample was increased to 192.

All participants were female university students enrolled in Portuguese and Brazilian academic institutions. The Portuguese sample 
consisted of 278 students enrolled in the University Institute of Psychological, Social, and Life Sciences (ISPA, $\mathrm{n}=104$ ), University of Algarve $(n=151)$, and Lisbon University Institute (ISCTE, $\mathrm{n}=23$ ). The Brazilian sample included 248 students enrolled at the Araraquara Campus of São Paulo State University (UNESP) in the city of Araraquara, São Paulo State. All participants were Humanities and Social Sciences majors.

All students were invited to participate in this study, but only those who gave their written consent were included. Inclusion criteria were age (> 18 years), sex (female), and enrollment in a university (in Portugal or Brazil).

Mean age in the overall sample was 20.9 years (standard deviation $[\mathrm{SD}]=1.4$ ). Mean age in the Portuguese sample was $20.9(\mathrm{SD}=2.4)$, and mean age of the Brazilian sample was 20.9 $(\mathrm{SD}=2.3)$. Mean overall body mass index $(\mathrm{BMI})$ was $22.7(\mathrm{SD}=2.4) \mathrm{kg} / \mathrm{m}^{2} ; 21.5(\mathrm{SD}=3.3)$ in the Portuguese sample and 22.7 (SD = 3.6) in the Brazilian sample. Table 1 shows the two samples' characteristics.

\section{Psychometric analysis of face validity}

To create the unified Portuguese-language version of the BSQ for use in both Brazil and Portugal, we used the Brazilian version proposed by Di Pietro \& Silveira 15, the Portuguese version proposed by Pimenta et al. 13, and the original version in English proposed by Cooper et al. 12 .

The three versions (Brazilian, Portuguese, and English) were analyzed by four researchers specialized in the area of body image and psychometrics (two Brazilian researchers and two Portuguese researchers) in order to create a unified Portuguese-language version that: (1) corresponded to the instrument's original version; and (2) would be clearly understood by Portuguese and Brazilian citizens alike. After obtaining agreement and consensus between researchers, the unified version was analyzed by a fourmember multidisciplinary team (including two Brazilian and two Portuguese experts in psychology and psychometrics). The team assessed the instrument's idiomatic, semantic, cultural, and conceptual equivalence. Once the final version was established, it was pretested (in a pilot study) in a sample of 30 university students (15 from each nationality) to determine the items' incomprehension index. The incomprehension index was used to assess participants' understanding of the items (including words and expressions). Suggestions for changing words or expressions in the items were written, and when incomprehension index was less than $20 \%$, the instrument was defined as displaying good comprehension. All the words were understood by the students, and no items were changed. Thus, the unified Portuguese-language version of the 34-item BSQ was adopted (Table 2) and applied to both samples.

\section{Instruments}

As mentioned previously, the 34-item unified Portuguese-language version of the BSQ was used. To assess concurrent validity of the BSQ, the Weight Concern Scale (WCS) was applied. The WCS was originally developed in English by Killen et al. 21 to assess body weight concerns among women. This scale was proposed as a one-factor model with 5 questions and was answered on a 7-point Likert-type response scale. The version of the WCS used in the present study was also a unified version (adapted for consistent use in both Brazil and Portugal), previously presented by Dias et al. 14. In the present study, data gathered using the WCS revealed good psychometric properties in the overall sample of Portuguese and Brazilian students $\left(\chi^{2} / \mathrm{df}=3.95\right.$, comparative fit index $[\mathrm{CFI}]=0.99$, normed fit index $[\mathrm{NFI}]=$ 0.98 , root mean square error of approximation $[$ RMSEA $]=0.07$, Cronbach's $\alpha=0.72$ ). Good psychometric properties were also found in the Portuguese sample alone $\left(\chi^{2} / \mathrm{df}=3.05, \mathrm{CFI}=0.98\right.$, $\mathrm{NFI}=0.97$, RMSEA $=0.08$, Cronbach's $\alpha=0.73$ ), as well as in the Brazilian sample alone $\left(\chi^{2} / \mathrm{df}=2.58\right.$, $\mathrm{CFI}=0.98$, NFI $=0.97$, RMSEA $=0.08$, Cronbach's $\alpha=0.73$ ).

A questionnaire was also applied to assert socio-demographic, weight-related, and academic characteristics.

\section{Procedures}

The research team contacted the universities to explain the project and to request the time necessary for students to complete the questionnaire (approximately 10 minutes). After receiving permission, research team members visited the classrooms at a time/day agreed on with the professors. After reading and signing an informed consent document, students present in the classrooms answered all the questionnaires (characterization questionnaire, BSQ, and WCS).

In Brazil, the study was approved by the Ethics Committee on Human Research at the Department of Pharmaceutical Sciences, UNESP (C.A.A.E.: 29896214.0.0000.5426). In Portugal, all aspects of the project (including the ethical aspects) were approved by the Ethics Committee of the research unit (Psychology and Health Research Unit) where the study was conducted. 
Demographic characteristics of the total, Brazilian, and Portuguese samples. Brazil, Portugal, 2014.

\begin{tabular}{|c|c|c|c|}
\hline \multirow[t]{2}{*}{ Characteristics } & \multicolumn{3}{|c|}{ Sample } \\
\hline & $\begin{array}{l}\text { Total } \\
\text { n (\%) }\end{array}$ & $\begin{array}{c}\text { Portugal } \\
\text { n (\%) }\end{array}$ & $\begin{array}{l}\text { Brazil } \\
\text { n (\%) }\end{array}$ \\
\hline \multicolumn{4}{|l|}{ Study shift } \\
\hline Mornings & $222(42.5)$ & $124(45.3)$ & 98 (39.5) \\
\hline Afternoons & $64(12.3)$ & $28(10.2)$ & $36(14.5)$ \\
\hline Nights & $102(19.5)$ & $17(6.2)$ & 85 (34.3) \\
\hline Fulltime & $134(25.7)$ & $105(38.3)$ & $29(11.7)$ \\
\hline \multicolumn{4}{|l|}{ Housing } \\
\hline Alone & $47(9.0)$ & $18(6.5)$ & $29(11.7)$ \\
\hline With family & $266(50.9)$ & $164(59.4)$ & $102(41.3)$ \\
\hline With friends or colleagues & $210(40.2)$ & $94(34.1)$ & $116(47.0)$ \\
\hline \multicolumn{4}{|l|}{ Expectation regarding degree } \\
\hline Worse & $47(9.0)$ & $16(5.8)$ & $31(12.6)$ \\
\hline Equal & $141(27.0)$ & $78(28.4)$ & $63(25.5)$ \\
\hline Better & $334(64.0)$ & $181(65.8)$ & $153(61.9)$ \\
\hline \multicolumn{4}{|l|}{ Student's performance } \\
\hline Excellent & $7(1.3)$ & $2(0.7)$ & $5(2.0)$ \\
\hline Good & $167(31.7)$ & $103(37.1)$ & $64(25.8)$ \\
\hline Average & $325(61.8)$ & $162(58.3)$ & $163(65.7)$ \\
\hline Bad & $27(5.1)$ & $11(4.0)$ & $16(6.5)$ \\
\hline \multicolumn{4}{|l|}{ Thoughts of giving up } \\
\hline Never & $136(25.9)$ & $8(2.9)$ & $128(51.6)$ \\
\hline Sometimes & $163(31.0)$ & $59(21.2)$ & $104(41.9)$ \\
\hline Frequently & $227(43.2)$ & $211(75.9)$ & $16(6.5)$ \\
\hline \multicolumn{4}{|l|}{ Have a job } \\
\hline No & $382(72.9)$ & $233(84.4)$ & $149(60.1)$ \\
\hline Yes & $142(27.1)$ & $43(15.6)$ & $99(39.9)$ \\
\hline \multicolumn{4}{|l|}{ Medication as study aid } \\
\hline Never & $392(74.8)$ & $210(75.5)$ & $182(74.0)$ \\
\hline Sometimes & $120(22.9)$ & $64(23.0)$ & $56(22.8)$ \\
\hline Frequently & $12(2.3)$ & $4(1.4)$ & $8(3.3)$ \\
\hline \multicolumn{4}{|l|}{ Parents' qualifications } \\
\hline Illiterate/incomplete primary school & $8(1.5)$ & - & $8(3.3)$ \\
\hline Complete primary school/incomplete middle school & $70(13.4)$ & $45(16.2)$ & $25(10.2)$ \\
\hline Complete middle school/incomplete high school & $99(18.9)$ & $71(25.6)$ & $28(11.4)$ \\
\hline Complete high school/incomplete college school & $169(32.3)$ & $82(29.6)$ & $87(35.4)$ \\
\hline Complete college school & $177(33.8)$ & $79(28.5)$ & $98(39.8)$ \\
\hline \multicolumn{4}{|l|}{ Nutritional status } \\
\hline Underweight & $30(5.7)$ & $23(8.3)$ & $7(2.8)$ \\
\hline Normal weight & $422(80.5)$ & $219(79.1)$ & $203(82.2)$ \\
\hline Overweight & $72(13.7)$ & 35 (12.6) & $37(15.0)$ \\
\hline
\end{tabular}


Original English and unified Portuguese-language version of the Body Shape Questionnaire (BSQ). Brazil, Portugal, 2014.

\begin{tabular}{ll}
\hline Item & Original English version \\
\hline 1 & Has feeling bored made you brood about your shape? \\
2 & Have you been so worried about your shape that you have been \\
3 & Have you thought that your thighs, hips, or bottom are too large for \\
4 & Have you been afraid that you might become fat (or fatter)? \\
5 & Have you worried about your flesh being not firm enough? \\
6 & Have you felt so bad about your shape that you have cried? \\
7 & Has being with thin women made you feel self-conscious about \\
9 & Have you worried about your thighs spreading out when sitting \\
10 & Has eating even a small amount of food made you feel fat?
\end{tabular}

Have you noticed the shape of other women and felt that your own shape compared unfavorably?

Has thinking about your shape interfered with your ability to concentrate (e.g. while watching television, reading, listening to conversations)?

Has being naked, such as when taking a bath, made you feel fat?

Have you avoided wearing clothes which make you particularly aware of the shape of your body?

Have you imagined cutting off fleshy areas of your body?

Has eating sweets, cakes, or other high calorie food made you feel fat?

Have you not gone out to social occasions (e.g. parties) because you have felt bad about your shape?

Have you felt excessively large and rounded?

Have you felt ashamed of your body?

Has worry about your shape made you diet?

Have you felt happiest about your shape when your stomach has been empty (e.g. in the morning)?

Have you thought that you are in the shape you are because you lack self-control?

Have you worried about other people seeing rolls of fat around your waist or stomach?

Have you felt that it is not fair that other women are thinner than you?

Have you vomited in order to feel thinner?

When in company have your worried about taking up too much room (e.g. sitting on a sofa, or a bus seat)?

Have you worried about your flesh being dimply?

Has seeing your reflection (e.g. in a mirror or shop window) made you feel bad about your shape?

Have you pinched areas of your body to see how much fat there is? Have you avoided situations where people could see your body

(e.g. communal changing rooms or swimming baths)?

Have you taken laxatives in order to feel thinner?

Have you been particularly self-conscious about your shape when in the company of other people?

Has worry about your shape made you feel you ought to exercise?

\section{Unified Portuguese-language version}

Ter-se sentido entediado(a) fez com que você se passasse a preocupar com a sua forma física?

Tem estado tão preocupado(a) com a forma do seu corpo que começou a pensar que deveria fazer dieta?

Já lhe ocorreu que as suas coxas, quadril/ancas ou nádegas são grandes demais em relação ao resto do seu corpo?

Tem sentido medo de ficar gordo(a) ou mais gordo(a)?

Preocupou-se com o seu corpo não ser firme o suficiente?

Sentir-se cheio(a) (por exemplo, depois de ingerir uma refeição grande) fez com que se sentisse gordo(a)?

Sentiu-se tão mal com a forma do seu corpo a ponto de chorar?

Evitou correr por achar que seu corpo poderia balançar?

Estar com pessoas magras, do mesmo sexo que o seu, faz com que se sinta desconfortável com a forma do seu corpo?

Preocupou-se com suas coxas ocuparem muito espaço quando se senta?

Comer, mesmo que uma pequena quantidade de comida, fez com que se sentisse gordo(a)?

Tem reparado na forma do corpo de outras pessoas do mesmo sexo que o seu e, ao comparar-se, sentiu-se em desvantagem?

Pensar na forma do seu corpo interferiu na sua capacidade de se concentrar noutras atividades (como por exemplo, ver televisão, ler ou acompanhar uma conversa)?

Estar nu(nua), por exemplo, durante o banho, fez com que se sentisse gordo(a)?

Já evitou usar roupas que o(a) façam reparar mais na forma do seu corpo?

Já imaginou remover (cortar) partes carnudas do seu corpo?

Comer doces, bolos e outros alimentos ricos em calorias fez com que se sentisse gordo(a)?

Deixou de ir a eventos sociais (como por exemplo, festas) por sentir-se mal com a forma do seu corpo?

Sentiu-se excessivamente grande e arredondado(a)? Sentiu vergonha do seu corpo?

A preocupação com a forma do seu corpo levou-o(a) a fazer dieta?

Sentiu-se mais contente em relação à forma do seu corpo quando seu estômago estava vazio (por exemplo, pela manhã)?

Acredita que a forma do seu corpo se deve à sua falta de autocontrole(o)?

Preocupou-se que outras pessoas vissem dobras na região da sua cintura ou estômago?

Pensou que não é justo que outras pessoas do mesmo sexo que o seu sejam mais magras que você?

Já vomitou para se sentir mais magro(a)?

Quando acompanhado(a), preocupou-se em ocupar um espaço excessivo (por exemplo, sentado(a) num sofá ou no banco de um transporte público)? Preocupou-se com o seu corpo estar com "pneus"?

Ver o seu reflexo (por exemplo, num espelho ou na vitrine de uma loja) fez com que se sentisse mal em relação ao seu corpo?

Beliscou áreas do seu corpo para ver a quantidade de gordura que existe?

Evitou situações nas quais as pessoas pudessem ver o seu corpo (por exemplo, vestiários)?

Já tomou laxantes para se sentir mais magro(a)?

Sentiu-se particularmente desconfortável com a forma do seu corpo, quando na companhia de outras pessoas?

A preocupação com a forma do seu corpo fez com que sentisse que deveria fazer exercício físico? 


\section{Psychometric analysis}

The psychometric properties of the unified Portuguese-language version were assessed in the overall sample, and also in the Portuguese and Brazilian samples separately.

\section{Psychometric sensitivity}

The scale items' psychometric sensitivity was determined by descriptive statistics (mean, median, mode, standard deviation, skewness, kurtosis). According to Maroco ${ }^{39}$, sensitivity is considered adequate when skewness has an absolute value of less than 3 and when kurtosis has an absolute value of less than 7. Multivariate outliers were identified using the Mahalanobis distance 40,41.

\section{Factorial validity}

Confirmatory factor analysis of the BSQ (34 items) was performed using maximum likelihood estimation. The goodness of fitness indices chi-square distribution with degrees of freedom $\left(\chi^{2} / \mathrm{df}\right)$, CFI, NFI, and RMSEA were used 39,41 . The model fit was considered adequate when $\lambda \geq 0.40$, $\chi^{2} / \mathrm{df} \leq 3.00$, CFI $\geq 0.90$, NFI $\geq 0.80$, and RMSEA $\leq 0.1039$. Modification indices estimated by the Lagrange Multipliers (LM) method were used to refine the model, and LM > 11 was adopted as the cutoff point 39 .

\section{Convergent validity}

Convergent validity was assessed according to Fornell \& Larcker's 42 proposal, which recommends calculation of the average variance extracted (AVE). AVE $\geq 0.50$ was considered evidence of convergent validity 39,40 .

A previous study 33 found that a shortened version of the BSQ (items 5, 11, 15, 20, 21, 22, 25, and 28) was a better model; data gathered with this version showed adequate validity and reliability when applied to a sample of Brazilian university students. Thus, we decided to also test the shortened model's fit for the total sample, as well as for the Brazilian and Portuguese samples.

To determine which model (34-item and shortened version) would be better, we calculated information theory indices, including the Bayesian information criterion (BIC), BrowneCudeck criterion (BCC), and Akaike information criterion (AIC). The model that showed the lowest values in all or most of the indices was considered the most suitable 39 .

\section{Concurrent validity}

Concurrent validity of the BSQ was assessed using Pearson Correlation Analysis (r) with the WCS.

\section{Reliability}

Reliability was estimated using internal consistency and composite reliability (CR). Internal consistency was calculated using the standardized Cronbach's $\alpha$ and was considered adequate when $\alpha \geq 0.7043$. CR was estimated according to Fornell \& Larcker 42 , and it was also considered adequate when $\mathrm{CR} \geq 0.7039,40$.

\section{Factorial invariance}

To establish the invariance of the factorial solution in the Brazilian and Portuguese samples, a multi-group cross-validation analysis was performed. First, the total sample was divided into two parts, one of which consisted of $60 \%$ of the total sample $(n=315)$ and was considered the test sample, and the second of which contained the other $40 \%(n=211)$ and was considered the validation sample. The invariance test was performed using multi-group analysis, which itself relied on the chi-square difference $\left(\Delta \chi^{2}\right)$ between the model with free factorial weights and the model with equal weights. If the hypothesis of factor loading invariance was accepted (metric invariance), then analyses of the invariance of intercepts (scalar invariance) and covariance of residuals (structural invariance) were also performed 39,44. The BSQ model's transnational invariance was also established. Its evaluation was performed as previously described - the Brazilian and Portuguese sub-samples were compared

\section{Data analysis}

Statistical analyses used IBM SPSS Statistics (v. 22, IMB Corp., Armonk, U.S.A.) and SPSS AMOS 22.0 (IBM Corp., Armonk, U.S.A.) software.

\section{Results}

Table 3 further outlines the BSQ items' characteristics. When psychometric sensitivity was considered, items 18, 26, 27, and 32 presented kurtosis and/or skewness values that differed from recommended values and were therefore inadequate in at least one of the samples (total, Portuguese and/or Brazilian sample).

Table 4 shows the BSQ's goodness of fit indices, average variance extracted, and reliability 
Table 3

Descriptive statistics for the Body Shape Questionnaire (BSQ) items for total, Portuguese, and Brazilian samples. Brazil, Portugal, 2014.

\begin{tabular}{|c|c|c|c|c|c|c|}
\hline \multirow[t]{2}{*}{ Item } & \multicolumn{6}{|c|}{ Total; Portuguese; Brazilian } \\
\hline & Mean & Median & Mode & Standard deviation & Skewness & Kurtosis \\
\hline 1 & $2.94 ; 2.90 ; 3.00$ & $3.00 ; 3.00 ; 3.00$ & $3.00 ; 3.00 ; 3.00$ & $1.30 ; 1.20 ; 1.40$ & $0.35 ; 0.14 ; 0.47$ & $-0.30 ;-0.50 ;-0.32$ \\
\hline 2 & $3.12 ; 2.87 ; 3.42$ & $3.00 ; 3.00 ; 3.00$ & $1.00 ; 1.00 ; 3.00$ & $1.65 ; 1.55 ; 1.72$ & $0.25 ; 0.32 ; 0.11$ & $-1.11 ;-1.04 ;-1.22$ \\
\hline 3 & $2.75 ; 2.78 ; 2.71$ & $2.00 ; 3.00 ; 2.00$ & $1.00 ; 1.00 ; 1.00$ & $1.73 ; 1.64 ; 1.83$ & $0.56 ; 0.45 ; 0.66$ & $-1.02 ;-1.04 ;-1.02$ \\
\hline 4 & $3.52 ; 3.22 ; 3.85$ & $3.00 ; 3.00 ; 4.00$ & $3.00 ; 3.00 ; 6.00$ & $1.68 ; 1.56 ; 1.75$ & $0.04 ; 0.20 ;-0.21$ & $-1.17 ;-0.96 ;-1.24$ \\
\hline 5 & $3.66 ; 3.50 ; 3.85$ & $3.00 ; 3.00 ; 4.00$ & $3.00 ; 3.00 ; 3.00$ & $1.50 ; 1.35 ; 1.63$ & $0.06 ; 0.14 ;-0.10$ & $-0.91 ;-0.61 ;-1.13$ \\
\hline 6 & $3.10 ; 2.79 ; 3.46$ & $3.00 ; 3.00 ; 3.00$ & $1.00 ; 1.00 ; 3.00$ & $1.67 ; 1.56 ; 1.73$ & $0.31 ; 0.52 ; 0.05$ & $-0.11 ;-0.80 ;-1.27$ \\
\hline 7 & $1.76 ; 1.65 ; 1.89$ & $1.00 ; 1.00 ; 1.00$ & $1.00 ; 1.00 ; 1.00$ & $1.26 ; 1.12 ; 1.38$ & $1.86 ; 1.96 ; 1.70$ & $2.87 ; 3.41 ; 2.12$ \\
\hline 8 & $1.46 ; 1.32 ; 1.62$ & $1.00 ; 1.00 ; 1.00$ & $1.00 ; 1.00 ; 1.00$ & $0.96 ; 0.71 ; 1.15$ & $2.46 ; 2.40 ; 2.10$ & $6.14 ; 5.59 ; 3.89$ \\
\hline 9 & $2.23 ; 2.13 ; 2.33$ & $2.00 ; 2.00 ; 2.00$ & $1.00 ; 1.00 ; 1.00$ & $1.43 ; 1.32 ; 1.54$ & $1.16 ; 1.16 ; 1.12$ & $0.57 ; 0.72 ; 0.27$ \\
\hline 10 & $2.02 ; 1.83 ; 2.23$ & $1.00 ; 1.00 ; 1.00$ & $1.00 ; 1.00 ; 1.00$ & $1.41 ; 1.23 ; 1.57$ & $1.36 ; 1.57 ; 1.12$ & $0.92 ; 1.84 ; 0.10$ \\
\hline 11 & $1.70 ; 1.60 ; 1.81$ & $1.00 ; 1.00 ; 1.00$ & $1.00 ; 1.00 ; 1.00$ & $1.11 ; 0.97 ; 1.24$ & $1.90 ; 1.97 ; 1.74$ & $3.55 ; 4.18 ; 2.62$ \\
\hline 12 & $2.94 ; 2.74 ; 3.15$ & $3.00 ; 3.00 ; 3.00$ & $3.00 ; 2.00 ; 3.00$ & $1.38 ; 1.26 ; 1.48$ & $0.60 ; 0.68 ; 0.46$ & $-0.34 ;-0.01 ;-0.68$ \\
\hline 13 & $1.63 ; 1.53 ; 1.79$ & $1.00 ; 1.00 ; 1.00$ & $1.00 ; 1.00 ; 1.00$ & $1.05 ; 0.85 ; 1.23$ & $1.90 ; 1.67 ; 1.75$ & $3.57 ; 2.32 ; 2.58$ \\
\hline 14 & $2.37 ; 2.17 ; 2.61$ & $2.00 ; 2.00 ; 2.00$ & $1.00 ; 1.00 ; 1.00$ & $1.48 ; 1.32 ; 1.60$ & $0.98 ; 1.15 ; 0.76$ & $0.04 ; 0.72 ;-0.54$ \\
\hline 15 & $3.01 ; 2.81 ; 3.21$ & $3.00 ; 3.00 ; 3.00$ & $3.00 ; 3.00 ; 3.00$ & $1.49 ; 1.40 ; 1.57$ & $0.44 ; 0.50 ; 0.33$ & $-0.65 ;-0.43 ;-0.88$ \\
\hline 16 & $2.21 ; 1.89 ; 2.56$ & $1.00 ; 1.00 ; 2.00$ & $1.00 ; 1.00 ; 1.00$ & $1.62 ; 1.36 ; 1.80$ & $1.13 ; 1.52 ; 0.76$ & $-0.01 ; 1.31 ;-0.86$ \\
\hline 17 & $2.83 ; 2.64 ; 3.04$ & $3.00 ; 2.00 ; 3.00$ & $1.00 ; 1.00 ; 1.00$ & $1.61 ; 1.47 ; 1.73$ & $0.63 ; 0.69 ; 0.50$ & $-0.68 ;-0.38 ;-1.01$ \\
\hline 18 & $1.41 ; 1.28 ; 1.55$ & $1.00 ; 1.00 ; 1.00$ & $1.00 ; 1.00 ; 1.00$ & $1.00 ; 0.74 ; 1.20$ & $2.90 ; 3.28 ; 2.41$ & $8.38 ; 12.29 ; 5.00$ \\
\hline 19 & $1.88 ; 1.73 ; 2.05$ & $1.00 ; 1.00 ; 1.00$ & $1.00 ; 1.00 ; 1.00$ & $1.33 ; 1.18 ; 1.47$ & $1.59 ; 1.78 ; 1.37$ & $1.72 ; 2.65 ; 0.87$ \\
\hline 20 & $2.42 ; 2.28 ; 2.58$ & $2.00 ; 2.00 ; 2.00$ & $2.00 ; 2.00 ; 2.00$ & $1.39 ; 1.33 ; 1.47$ & $0.96 ; 1.09 ; 0.83$ & $0.15 ; 0.51 ;-0.14$ \\
\hline 21 & $2.70 ; 2.57 ; 2.85$ & $2.00 ; 2.00 ; 3.00$ & $1.00 ; 1.00 ; 1.00$ & $1.66 ; 1.68 ; 1.63$ & $0.55 ; 0.64 ; 0.48$ & $-0.96 ;-0.95 ;-0.91$ \\
\hline 22 & $3.04 ; 2.88 ; 3.22$ & $3.00 ; 3.00 ; 3.00$ & $1.00 ; 1.00 ; 1.00$ & $1.84 ; 1.79 ; 1.89$ & $0.35 ; 0.42 ; 0.24$ & $-1.30 ;-1.17 ;-1.41$ \\
\hline 23 & $2.80 ; 2.63 ; 2.99$ & $3.00 ; 2.00 ; 3.00$ & $1.00 ; 1.00 ; 1.00$ & $1.68 ; 1.60 ; 1.75$ & $0.57 ; 0.72 ; 0.40$ & $-0.89 ;-0.63 ;-1.10$ \\
\hline 24 & $2.86 ; 2.61 ; 3.15$ & $3.00 ; 2.00 ; 3.00$ & $1.00 ; 1.00 ; 1.00$ & $1.62 ; 1.53 ; 1.68$ & $0.47 ; 0.62 ; 0.30$ & $-0.88 ;-0.65 ;-1.07$ \\
\hline 25 & $1.86 ; 1.79 ; 1.95$ & $1.00 ; 1.00 ; 1.00$ & $1.00 ; 1.00 ; 1.00$ & $1.34 ; 1.26 ; 1.43$ & $1.68 ; 1.76 ; 1.59$ & $2.01 ; 2.49 ; 1.55$ \\
\hline 26 & $1.15 ; 1.13 ; 1.17$ & $1.00 ; 1.00 ; 1.00$ & $1.00 ; 1.00 ; 1.00$ & $0.62 ; 0.59 ; 0.66$ & $5.35 ; 5.61 ; 5.11$ & $31.64 ; 34.18 ; 29.66$ \\
\hline 27 & $1.42 ; 1.38 ; 1.46$ & $1.00 ; 1.00 ; 1.00$ & $1.00 ; 1.00 ; 1.00$ & $0.97 ; 0.90 ; 1.03$ & $2.83 ; 2.93 ; 2.73$ & $8.37 ; 9.32 ; 7.50$ \\
\hline 28 & $2.69 ; 2.42 ; 2.99$ & $2.00 ; 2.00 ; 3.00$ & $1.00 ; 1.00 ; 1.00$ & $1.52 ; 1.35 ; 1.64$ & $0.68 ; 0.82 ; 0.46$ & $-0.48 ;-0.08 ;-0.89$ \\
\hline 29 & $2.46 ; 2.28 ; 2.67$ & $2.00 ; 2.00 ; 2.00$ & $1.00 ; 1.00 ; 1.00$ & $1.41 ; 1.30 ; 1.50$ & $0.89 ; 0.96 ; 0.77$ & $0.04 ; 0.31 ;-0.30$ \\
\hline 30 & $2.93 ; 2.65 ; 3.24$ & $3.00 ; 3.00 ; 3.00$ & $3.00 ; 3.00 ; 2.00$ & $1.53 ; 1.35 ; 1.65$ & $0.50 ; 0.56 ; 0.31$ & $-0.67 ;-0.31 ;-1.08$ \\
\hline 31 & $2.34 ; 2.07 ; 2.65$ & $2.00 ; 2.00 ; 2.00$ & $1.00 ; 1.00 ; 1.00$ & $1.50 ; 1.26 ; 1.66$ & $1.08 ; 1.27 ; 0.83$ & $0.28 ; 1.16 ;-0.50$ \\
\hline 32 & $1.21 ; 1.14 ; 1.30$ & $1.00 ; 1.00 ; 1.00$ & $1.00 ; 1.00 ; 1.00$ & $0.77 ; 0.63 ; 0.89$ & $4.30 ; 5.60 ; 3.50$ & $19.64 ; 34.45 ; 12.63$ \\
\hline 33 & $2.35 ; 2.14 ; 2.60$ & $2.00 ; 2.00 ; 2.00$ & $1.00 ; 1.00 ; 2.00$ & $1.37 ; 1.23 ; 1.47$ & $0.99 ; 1.09 ; 0.83$ & $0.30 ; 0.69 ;-0.15$ \\
\hline 34 & $4.87 ; 3.69 ; 4.08$ & $4.00 ; 4.00 ; 4.00$ & $6.00 ; 3.00 ; 6.00$ & $1.58 ; 1.55 ; 1.58$ & $-0.19 ;-0.10 ;-0.32$ & $-1.04 ; 1.05 ;-0.98$ \\
\hline
\end{tabular}

for the unified version (34-item), refined version (32-item), and shortened version (8-item). These models were applied to the total sample and to the Brazilian and Portuguese samples individually. The complete model showed unsatisfactory fit for the total sample, so a refinement was performed. Items 26 and 32 were excluded due to their lower factor weights $(<0.40)$. Correlations were inserted between errors of items 24 and 28, 3 and 10, 2 and 21, 13 and 18, 6 and 22, 2 and 4, 20 and 23, 18 and 19, 12 and 33, 10 and 27, 7 and 23, 4 and 20, and 1 and 3. After these changes, the refined model showed satisfactory goodness of fit for all three samples (total, Brazilian, and Portuguese). The shortened model was found to be a good fit for all three samples as well.

When we considered the goodness of fit of the analyzed versions and the indices based on information theory (BCC, AIC, BIC), the short 8-item version of BSQ was found to be better. Reliability (CR and $\alpha$ ) was also considered, and all models presented appropriate values for all samples tested. 
Table 4

Psychometric characteristics of the complete, refined, and shortened versions of the Body Shape Questionnaire (BSQ) used on both Brazilian and Portuguese students. Brazil, Portugal, 2014.

\begin{tabular}{|c|c|c|c|c|c|c|c|c|c|c|c|}
\hline \multirow[t]{2}{*}{ Model * } & \multicolumn{5}{|c|}{ CFA } & \multirow[t]{2}{*}{ AVE } & \multirow[t]{2}{*}{ CR } & \multirow[t]{2}{*}{$\alpha$} & \multirow[t]{2}{*}{$\mathrm{BIC}$} & \multirow[t]{2}{*}{$\mathrm{BCC}$} & \multirow[t]{2}{*}{ AIC } \\
\hline & $\lambda$ & $\chi 2 / \mathrm{df}$ & CFI & NFI & RMSEA & & & & & & \\
\hline \multicolumn{12}{|l|}{ 34-item BSQ (complete) } \\
\hline Total sample & $0.34-0.87$ & 5.63 & 0.81 & 0.78 & 0.09 & 0.48 & 0.97 & 0.97 & $3,393.23$ & $3,112.91$ & $3,103.20$ \\
\hline \multicolumn{12}{|l|}{ 32-item BSQ (refined) } \\
\hline Total sample & $0.49-0.87$ & 4.10 & 0.88 & 0.86 & 0.07 & 0.50 & 0.97 & 0.97 & $2,332.55$ & $2,014.45$ & $2,004.12$ \\
\hline Brazilian sample & $0.43-0.89$ & 2.87 & 0.86 & 0.81 & 0.08 & 0.51 & 0.97 & 0.97 & $1,720.87$ & $1,474.09$ & $1,450.34$ \\
\hline Portuguese sample & $0.40-0.84$ & 2.65 & 0.88 & 0.82 & 0.07 & 0.50 & 0.97 & 0.97 & $1,628.25$ & $1,369.75$ & $1,348.92$ \\
\hline \multicolumn{12}{|l|}{ 8-item BSQ (shortened) } \\
\hline Total sample & $0.64-0.76$ & 5.01 & 0.95 & 0.94 & 0.08 & 0.50 & 0.88 & 0.88 & 200.49 & 132.81 & 132.25 \\
\hline Brazilian sample & $0.65-0.78$ & 3.25 & 0.95 & 0.92 & 0.09 & 0.50 & 0.88 & 0.88 & 153.18 & 98.17 & 96.97 \\
\hline Portuguese sample & $0.66-0.76$ & 3.08 & 0.95 & 0.93 & 0.08 & 0.50 & 0.88 & 0.87 & 151.68 & 94.71 & 93.63 \\
\hline
\end{tabular}

$\alpha$ : Cronbach's alpha; $\lambda$ : factorial weight; $\chi^{2 / d f: ~ c h i-s q u a r e ~ b y ~ d e g r e e s ~ o f ~ f r e e d o m ; ~ A I C: ~ A k a i k e ~ i n f o r m a t i o n ~ c r i t e r i o n ; ~ A V E: ~ a v e r a g e ~ v a r i a n c e ~ e x t r a c t e d ; ~ B C C: ~}$

Browne-Cudeck criterion; BIC: Bayes information criterion; CFA: confirmatory factor analysis; CFI: comparative fit index; CR:

composite reliability; NFI: normed fit index; RMSEA: root mean square error of approximation.

* Total sample: $N=526$; Brazilian Sample: $n=248$; Portuguese sample: $n=278$.

Correlational analysis between BSQ and WCS showed that all BSQ models (refined and shortened) were strongly correlated with WCS in the total (refined: $\mathrm{r}=0.756, \mathrm{p}<0.001$; shortened: $\mathrm{r}=$ 0.764, $\mathrm{p}<0.001$ ) Portuguese (refined: $\mathrm{r}=0.784$, $\mathrm{p}<0.001$; shortened: $\mathrm{r} 0.786, \mathrm{p}<0.001$ ), and Brazilian samples (refined: $\mathrm{r}=0.759, \mathrm{p}<0.001$; shortened: $r$ 0.762, $p<0.001$ ). This result shows the adequate concurrent validity of the data gathered with the BSQ and WCS.

When exploring the factorial invariance, we observed that the refined model (32-item) presented metric, scalar, and structural invariance in independent samples $\left(\Delta \chi^{2} \lambda(31)=33.18, \mathrm{p}=0.361\right.$; $\Delta \chi^{2}$ Intercepts $(63)=70.15, \mathrm{p}=0.250 ; \Delta \chi^{2}$ Covariance $(64)=72.22, p=0.225$ ), and the shortened model presented metric, scalar, and structural measure invariance $\left(\Delta \chi_{\lambda}^{2}{ }_{\lambda}(7)=2.60, \mathrm{p}=0.920 ; \Delta \chi^{2}\right.$ Intercepts $(15)=9.54, \mathrm{p}=0.858 ; \Delta \chi^{2}$ Covariance $(16)=10.97, \mathrm{p}=$ 0.811 ). When transnational invariance (Brazilian vs. Portuguese) was considered, the refined model was not stable between samples from the two countries $\left(\Delta \chi^{2}{ }_{\lambda}(31)=95.06, \mathrm{p}<0.001 ; \Delta \chi^{2}\right.$ Intercepts $(63)=198.00 \mathrm{p}<0.001 ; \Delta \chi^{2}$ Covariance $(64)=207.48$, $\mathrm{p}<0.001)$. The shortened model presented metric measure invariance $\left(\Delta \chi^{2} \lambda(7)=11.32, \mathrm{p}=0.125\right.$; $\Delta \chi^{2}$ Intercepts $(15)=32.81, \mathrm{p}=0.005 ; \Delta \chi^{2}$ Covariance (16) $=36.94, p=0.002$ ). Figure 1 shows the analysis of the shortened BSQ models used on the Portuguese and the Brazilian samples.

\section{Discussion}

The current study presents a unified Portugueselanguage version of the BSQ that can be used in both Brazilian and Portuguese research and clinical contexts. The psychometric properties of the data gathered in the samples using this version of the BSQ were assessed and are considered adequate for studies in Brazilian and Portuguese samples of female university students. Use of this version to evaluate body shape concerns in transnational studies (in Brazil and Portugal) can thus be considered feasible and should promote further collaborative studies including both populations.

In order to improve the complete model's goodness of fit when using the total sample (Portuguese and Brazilian students), items 26 and 32 were removed. The exclusion of these two items has also been proposed in previous studies using non-clinical samples 13,33,45. Usually, studies with normative samples tend to exclude these items, since they present low factor weight. This occurs because the content of the items refers to purging behaviors, which are only observed in people with severe eating disorders.

In order to further improve the refined BSQ model's goodness of fit, we also had to implement correlations between errors in the items. This strategy is used when the contents of two or more given items present similarities. We found, 
Confirmatory factor analysis of the shortened models of the Body Shape Questionnaire (BSO) used in the Brazilian and

Portuguese samples. Brazil, Portugal, 2014.

1a) Brazilian model

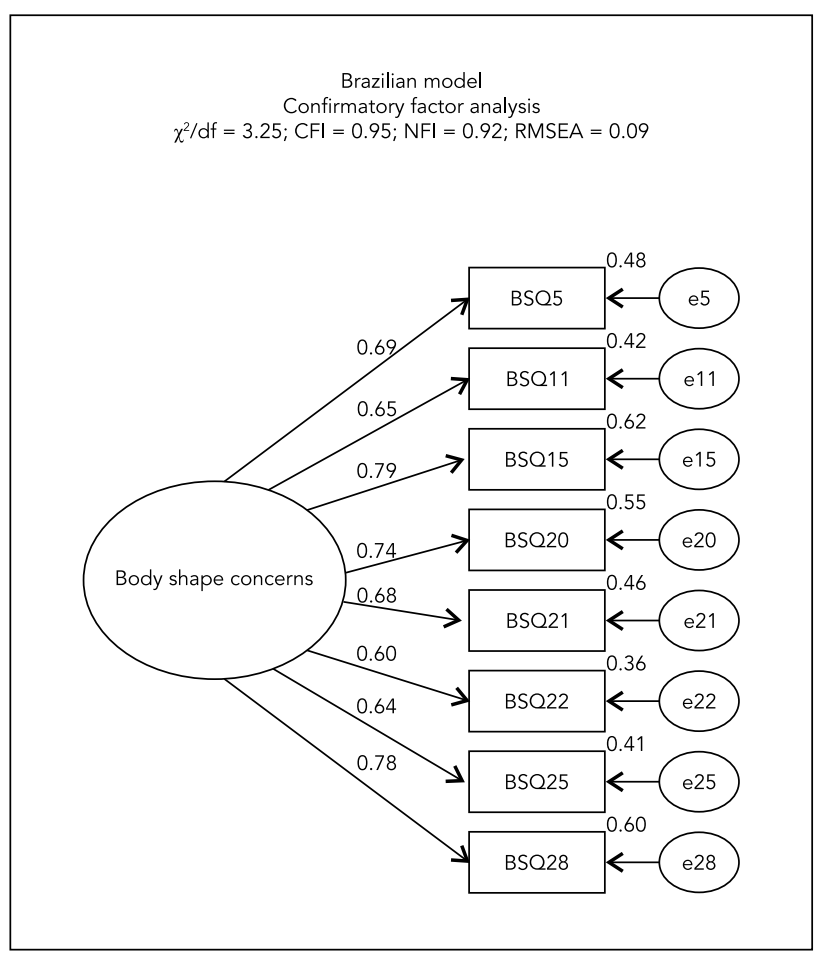

1b) Portuguese model

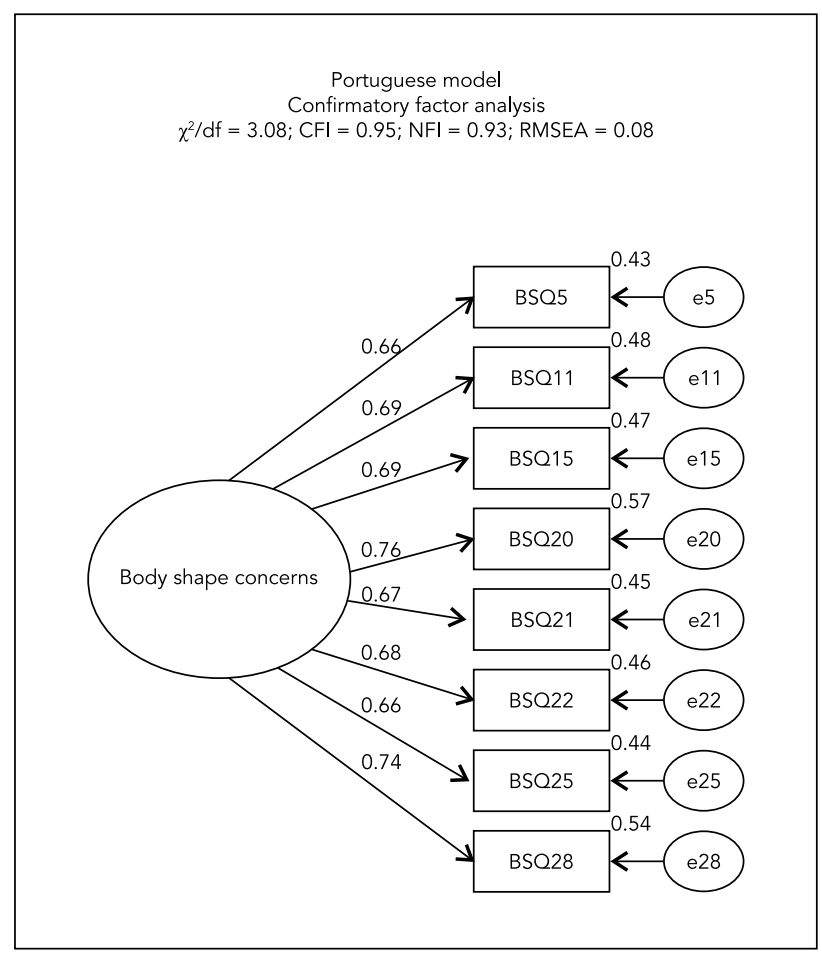

$\chi 2 / d f$ : chi-square by degrees of freedom; CFI: comparative fit index; NFI: normed fit index; RMSEA: root mean square error of approximation.

for example, that correlations between errors 2 and 21 (both focusing on dieting behaviors subsequent to body shape concern) and 24 and 28 (both entailing concerns regarding flesh, i.e., rolls of flesh or the flesh being dimply) have also been reported in a previous study by Da Silva et al. 33. The fact that these correlations had previously appeared in a study on a Brazilian sample suggests that some cultural aspects are similar between the two countries, and these consistent results therefore support the validity of our transcultural BSQ version.

All three samples (total, Brazilian, and Portuguese) and all three models (complete 34 -item questionnaire, refined 32-item questionnaire, and shortened 8-item questionnaire) displayed satisfactory goodness of fit based on their validity and reliability estimates. These results are consistent with the literature $13,30,33,45,46$.
An adequate concurrent validity of the BSQ with the WCS has been asserted in the present study; this validity was also noted in the previous study by Da Silva et al. 33 using the BSQ with the WCS on a Brazilian sample.

The present research also emphasizes that the shortened model (8-items) is more efficient than the refined model (32-items); this finding has been shown in earlier studies 33,45 .

In addition, our results reveal the refined and shortened models' metric, scalar, and structural invariance for independent samples, indicating the model's invariance. We also found that the refined model is not invariant between the Brazilian and the Portuguese samples, but the shortened model presented metric invariance between both groups, confirming the model's transnational invariance. The BSQ-8 can thus measure the same construct consistently within the two cultures. 
The unified Portuguese-language version presented in this study and tested on two independent and culturally different samples (one from Brazil and the other from Portugal) possesses adequate psychometric properties when used in a non-clinical sample of female Brazilian and Portuguese university students. This is true for both the refined and shortened models. However, we suggest that the use of the short-

\section{Contributors}

W. R. Silva participated in the study's conceptualization and design, data collection and analysis, and writing of all versions of the article. D. Costa participated in the study's conceptualization and design, data analysis, writing of all versions of the article. F. Pimenta, J. Maroco and J. A. D. B. Campos participated in the study's conceptualization and design, data analysis, and critical revision of the article. ened model is more adequate, since it presented goodness of fit without correlations or exclusions of items and was stable in both independent and transnational samples. Thus, this study represents a first step into cross-cultural research in the area of body image. The unified version of this instrument presented herein may increase the strength of the transcultural evidence on body shape concerns.

\section{Acknowledgments}

We are grateful to psychologist Fernanda Cristina Maurício (CRP 06/119177) for the data collection in the Brazilian sample and Mafalda Leitão, Adriana Bernardino, and Patrícia Ferreira for the data collection in the Portuguese sample. FAPESP (Grant \#2014/03093-2 and 2015/00228-7).

\section{References}

1. Cash TF, Pruzinsky T. Future challenges for body image theory, research, and clinical, practice. In: Cash TF, Pruzinsky, editors. Body image: a handbook of theory, research, and clinical practice. New York: Guilford Press; 2002. p. 509-16.

2. Gallagher S, Cole J. Body image and body schema in a deafferented subject. Journal of Mind and Behavior 1995; 16:369-89.

3. Longo M, Azanon E, Haggard P. More than skin deep: body representation beyond primary somatosensory cortex. Neuropsychologia 2010; 48:655-68.

4. Woertman L, Brink F. Body image and female sexual functioning and behavior: a review. J Sex Res 2012; 49:184-211.
5. Cash TF, Smolak L. Crucial considerations in the assessment of body image. In: Press G, editor. Body image: a handbook of science, practice, and prevention. New York: Guilford Press; 2011. p. 369-77.

6. Campana ANNB, Tavares MCGCF, Garcia Júnior C. Preocupação e insatisfação com o corpo, checagem e evitação corporal em pessoas com transtornos alimentares. Paidéia (Ribeirão Preto) 2012; 22:375-81.

7. Cash TF, Fleming EC. The impact of body image experiences: development of the body image quality of life inventory. Int J Eat Disord 2002; 31: 455-60. 
8. Johnson F, Wardle J. Dietary restraint, body dissatisfaction, and psychological distress: a prospective analysis. J Abnorm Psychol 2005; 114:119-25.

9. Neumark-Sztianer D, Paxton S, Hannan P, Haines J, Story M. Does body satisfaction matter? Five-year longitudinal associations between body satisfaction and health behaviors in adolescent females and males. J Adolesc Health 2006; 39:244-51.

10. Paxton S, Neumark-Sztianer D, Hannan P. Body dissatisfaction prospectively predicts depressive mood and low self-esteem in adolescent girls and boys. J Clin Child Adolesc Psychol 2006; 35:539-49.

11. Tiggemann M. Television and adolescent body image: the role of program content and viewing motivation. J Soc Clin Psychol 2005; 24:361-81.

12. Cooper PJ, Taylor MJ, Cooper Z, Fairburn CG. The development and validation of the Body Shape Questionnaire. Int J Eat Disord 1987; 6:485-94.

13. Pimenta F, Leal I, Maroco J, Rosa B. Validação do Body Shape Questionnaire (BSQ) numa amostra de mulheres de meia-idade. In: Actas do 9o Congresso Nacional de Psicologia da Saúde. Aveiro: Placebo; 2012. p. 1139-44.

14. Dias JCR, Silva WR, Maroco J, Campos JADB. Validation of the Weight Concerns Scale applied to Brazilian university students. Body Image 2015; 30:72-6.

15. Di Pietro M, Silveira DX. Internal validity, dimensionality and performance of the Body Shape Questionnaire in a group of Brazilian college students. Rev Bras Psiquiatr 2009; 31:21-4.

16. Carvalho PHB, Ferreira MEC, Kotait M, Teixeira PC, Hearst N, Cordás TA, et al. Equivalências conceitual, semântica e instrumental: análises preliminares da versão em português (Brasil) da Male Body Dissatisfaction Scale (MBDS). Cad Saúde Pública 2013; 29:403-9.

17. Thompson JK, Coovert MD, Stormer S. Body image, social comparison, and eating disturbance: a covariance structure modeling investigation. Int J Eat Disord 1999; 26:43-51.

18. Alvarenga MS, Philippi ST, Lourenço BH, Sato PM, Scagliusi FB. Insatisfação com a imagem corporal em universitárias brasileiras. J Bras Psiquiatr 2010; 59:44-51.

19. Cooley E, Toray T. Body image and personality predictors of eating disorder symptoms during the college years. Int J Eat Disord 2001; 30:28-36.

20. Kakeshita IS, Silva AIP, Zanatta DP, Almeida SS. Construção e fidedignidade teste-reteste de escalas de silhuetas brasileiras para adultos e crianças. Psicol Teor Pesqui 2009; 25:263-70.

21. Killen JD, Taylor CB, Hayward C, Wilson DM, Haydel KF, Hammer LD, et al. Pursuit of thinness and onset of eating disorder symptoms in a community sample of adolescent girls: a three-year prospective analysis. Int J Eat Disord 1994; 16: 227-38.

22. Ochner CN, Gray JG, Brickner K. The development and initial validation of a new measure of male body dissatisfaction. Eat Behav 2009; 10:197-201.

23. Zabinski M, Pung M, Wilfley D, Eppstein D, Winzelberg A, Celio A, et al. Reducing risk factors for eating disorders: targeting at-risk women with a computerized psychoeducational program. Int J Eat Disord 2001; 29:401-8.
24. Moreno DC, Montaño IL, Prieto GA, Pérez-Acosta AM. Validación del Body Shape Questionnaire Cuestionario de la figura corporal) BSQ para la población colombiana. Acta Colomb Psicol 2007; 10:15-23.

25. Dowson J, Henderson L. The validity of a short version of the Body Shape Questionnaire. Psychiatry Res 2001; 102:263-71.

26. Rousseau A, Knotter A, Barbe P, Raich RM, Chabrol $\mathrm{H}$. Étude de validation de la version française du Body Shape Questionnaire. Encéphale 2005; 31:162-73.

27. Akdemir A, Inandi T, Akbas D, Kahilogullari AK, Eren M, Canpolat BI. Validity and reliability of a Turkish version of the Body Shape Questionnaire among female high school students: preliminary examination. Eur Eat Disord Rev 2012; 20:114-5.

28. Alcaraz SGO, Caballero LV, Rodríguez NEM, Ayensa JIB. Validación y confiabilidad del Body Shape Questionnaire (BSQ) en adolescentes de la ciudad de Colima. Revista Científica Electrónica de Psicología 2007; 4:204-16.

29. Raich RM, Mora M, Soler A, Avila C, Clos I, Zapater L. Adaptación de un instrumento de evaluación de la insatisfacción corporal. Clín Salud 1996; (1): 51-66.

30. Warren CS, Cepeda-Benito A, Gleaves DH, Moreno S, Rodriguez S, Fernandez MC, et al. English and Spanish versions of the Body Shape Questionnaire: measurement equivalence across ethnicity and clinical status. Int J Eat Disord 2008; 41:265-72.

31. Ghaderi A, Scott B. The reliability and validity of the Swedish version of the Body Shape Questionnaire. Scand J Psychol 2004; 45:319-24.

32. Cordás TA, Castilho S. Body image on the eating disorders - evaluation instruments: "Body Shape Questionnaire". Psiquiatr Biol 1994; 2:17-21.

33. Da Silva WR, Dias JCR, Maroco J, Campos JADB. Confirmatory factor analysis of different versions of the Body Shape Questionnaire applied to Brazilian university students. Body Image 2014; 11:38490.

34. Campos JADB, Maroco J. Adaptação transcultural Portugal-Brasil do Inventário de Burnout de Maslach para estudantes. Rev Saúde Pública 2012; 46:816-24.

35. De Bolle M, De Fruyt F, McCrae RR, Löckenhoff CE, Costa Jr. PT, Aguilar-Vafaie ME, et al. The emergence of sex differences in personality traits in early adolescence: a cross-sectional, cross-cultural study. J Pers Soc Psychol 2015; 108:171-85.

36. Bosi MLM, Luiz RR, Uchimura KY, Oliveira FP. Comportamento alimentar e imagem corporalentre estudantes de educação física. J Bras Psiquiatr 2008; 27:28-33.

37. Branco LM, Hilário MOE, Cintra IP. Percepção e satisfação corporal em adolescentes e a relação com seu estado nutricional. Arch Clin Psychiatry (São Paulo, Impr.) 2006; 33:292-6.

38. Kim KH. The relation among fit indexes, power and sample size in structural equation modeling. Struct Equ Modeling 2005; 12:368-90.

39. Maroco J. Análise de equações estruturais. Lisboa: Report Number; 2014. 
40. Hair JF, Black WC, Babin B, Anderson RE, Tatham RL. Multivariate data analysis. $6^{\text {th }}$ Ed. Upper Saddle River: Pearson Prentice Hall; 2005.

41. Byrne BM. Structural equation modeling with Amos: basic concepts, applications and programming. New Jersey: Lawrence Erlbaum Associates; 2001.

42. Fornell C, Larcker DF. Evaluating structural equation models with unobservable variables and measurement error. J Mark Res 1981; 18:39-50.

43. Maroco J, Garcia-Marques T. Qual a fiabilidade do alfa de Cronbach? Questões antigas e soluções modernas? Laboratório de Psicologia 2006; 4 : 65-90.

\section{Resumo}

Os objetivos do estudo foram desenvolver uma versão unificada em língua portuguesa do Body Shape Questionnaire (BSQ) e estimar sua validade, confiabilidade e consistência interna entre estudantes universitárias no Brasil e em Portugal. Foi realizada análise fatorial confirmatória utilizando a versão original (34 itens) $e$ abreviada (8 itens) do questionário. O ajuste do modelo foi avaliado com $\chi^{2} / g l$, CFI, NFI e RMSEA. O estudo avaliou a validade concorrente e convergente. A confiabilidade foi estimada por meio da consistência interna e confiabilidade composta ( $\alpha$ ). A invariância do BSQ foi testada por análise de múltigrupos. O modelo original de 32 itens foi refinado para apresentar melhor ajuste e validade e confiabilidade adequadas. O modelo breve mostrou ser estável nas duas amostras independentes e em amostras transnacionais (Brasil e Portugal). O uso da versão unificada é recomendado para a avaliação de questões de imagem corporal em estudantes universitárias brasileiras e portuguesas.

Psicometria; Imagem Corporal; Reprodutibilidade dos Testes; Inquéritos e Questionários
44. Kaplan D. Structural equation modeling: foundations and extensions. Thousand Oaks: Sage Publications; 2000.

45. Evans C, Dolan B. Body Shape Questionnaire: derivation of shortened "alternate forms". Int J Eat Disord 1993; 13:315-21.

46. Pook M, Tuschen-Caffier B, Brähler E. Evaluation and comparison of different versions of the Body Shape Questionnaire. Psychiatry Res 2008; 158 : 67-73.

\section{Resumen}

El objetivo de este estudio ha sido desarrollar una versión unificada en lengua portuguesa -entre Brasil y Portugal- del Body Shape Questionnaire (BSQ), y estimar su validez, fiabilidad, y consistencia interna en estudiantes universitarias brasileñas y portuguesas. El análisis factorial confirmatorio fue realizado usando tanto la versión original (34-ítems), como la reducida (8-ítems). El ajuste del modelo fue evaluado por los indices $\chi^{2} / d f$, CFI, NFI y RMSEA. También se evaluó la validez concurrente y convergente. La fiabilidad se estimó a través de la consistencia interna y fiabilidad compuesta ( $\alpha$ ). La invariancia transnacional del BSQ se probó utilizando análisis multigrupo. El modelo original fue redefinido (32 items) para que presentara un mejor ajuste, así como una validez y fiabilidad adecuadas. El modelo reducido era estable, tanto en muestras independientes, como en las transnacionales (Brasily Portugal). El uso de esta versión unificada se recomienda para la evaluación de aspectos relacionados con el cuestionario de figura corporal, tanto en estudiantes universitarias brasileñas, como portuguesas.

Psicometría; Imagen Corporal; Reproducibilidad de Resultados; Encuestas y Cuestionarios
Submitted on 18/Aug/2015

Final version resubmitted on 16/Dec/2015

Approved on 25/Feb/2016 